DOI 10.18699/PlantGen2019-086

\title{
Evaluation of genetic diversity of Fagopyrum esculentum Moench variety using method of ISSR-analysis
}

\author{
Klykov A.G.*, Chibizova A.S., Fisenko P.V., Barsukova E.N. \\ Federal Scientific Center of Agricultural Biotechnology of the Far East named after A.K. Chaika, \\ Ussuriysk, Russia \\ *e-mail:alex.klykov@mail.ru
}

Species of the genus Fagopyrum Mill. are prospective sources of flavonoids, the main among which is the 3-O-rutinoside quercetin. The most important task of selection is to develop new varieties ones with a high content of flavonoids. The study of intraspecific genetic diversity of $F$. esculentum is extremely relevant, due to the presence of a large number of the variety, which differ in the content of flavonoids. In this regard, the aim of this study was to evaluate the genetic polymorphism of the buckwheat variety of different origin with a high content of flavonoids using the molecular marking. Using method of ISSR-analysis there were investigated 5 varieties of $F$. esculentum: Izumrud (Primorsky Krai, Russia), Kitawase 1 (Japan), Kitawase 2 (Japan), Cheremshanka (Tatarstan, Russia), Bashkirskaya with red stem (Bashkortostan, Russia), using four primers. As a result of the PCR there were revealed 106 amplicons, 105 of which were polymorphic. The polymorphism (P) in the joined sample was $99.6 \%$. However, the variability within the varieties significantly differs from the minimum $\mathrm{P}=50$ and $50.94 \%$ in Izumrud and Bashkirskaya with red stem accordingly, to the maximum $\mathrm{P}=75.47 \%$ in Cheremshanka. Varieties Kitawase 1 and Kitawase 2 have close values of polymorphism $\mathrm{P}=65.09$ and $64.15 \%$, respectively. Based on the analysis of the distribution pattern of the revealed fragments, there were calculated the indices of differences - the genetic distances $\left(D_{N}\right)$. The highest value of $\mathrm{D}_{\mathrm{N}}=0.2296$ were defined between the varieties Izumrud and Bashkirskaya with red stem, the smallest $D_{N}=0.0284$ was found between Kitawase 1 and Kitawase 2. Varieties Izumrud, Bashkirskaya with red-stem and Kitawase 1, which have the greatest genetic differences, present the practical interest for the development of new genotypes with a high content of flavonoids.

Acknowledgements: The work is fulfilled in the frame of the programme "The Priority Scientific Research in favour of the complex development of the Far Eastern Branch of the Russian Academy of Sciences". 